\title{
Therapeutic Electrical Stimulation Currents in Chronic Non-specific Low Back Pain: Designing a Systematic Review
}

\author{
S. Izadi Laybidy 1,2, T. Shahmahmoodi 1,2, A. Rahimi ${ }^{3}$, F. Bokaee', A. A. Pourmomeny', \\ Z. S. Rezaeian ${ }^{1}$ \\ 1 Musculoskeletal Research Center and Department of Physical Therapy, Faculty of Rehabilitation Sciences, \\ Isfahan University of Medical Sciences, Isfahan, Iran \\ 2 Student Research Committee of Rehabilitation Students (Treata), Faculty of Rehabilitation Sciences, Isfahan \\ University of Medical Sciences, Isfahan, Iran \\ 3 Health Information Research Center, Head of Clinical Informationist Research Group, Faculty of Management \\ and Medical Information Sciences, Isfahan University of Medical Sciences, Isfahan, Iran
}

\section{CORRESPONDING AUTHOR: \\ Zahra Sadat Rezaeian \\ Musculoskeletal Research Center \\ Rehabilitation Sciences Research Institute \\ Department of Physical Therapy \\ Faculty of Rehabilitation Sciences \\ Isfahan University of Medical Sciences \\ Hezar Jerib Street 2 \\ Isfahan, Iran 8174673461 \\ E-mail: zrezaeian@rehab.mui.ac.ir}

DOI:

10.32098/mltj.03.2020.19

LEVEL OF EVIDENCE: $1 \mathrm{~A}$

\begin{abstract}
SUMMARY
Introduction. The aim of this study will be to evaluate the effect of electrical stimulation currents on the pain and function in subjects with chronic non-specific low back pain. Methods. Pubmed, ISI Web of Science, Scopus, Clinical Key, Science Direct, Medline, Embase, PEDro, ProQuest, the Cochrane Library, PROSPERO, and also the MOH Thesis, MOH Articles, Magiran, and SID as the national databases will be searched. Also, Google Scholar search engine will be used. All study types except Qualitative Studies, and Narrative Reviews, i.e. Clinical Trials, Cohort, Case-controls, Cross-sectionals, Observational Descriptive, Case Report, Case Series, Ecological Studies, Systematic Reviews, thesis and dissertation, in English and Persian will be retrieved. The publication date should not be after August 2019. To ensure all the publication has been reached, search will be extended to three years before the publication date of first article found for each type of electrical stimulation currents. The search will be limited to human studies of subjects between 18-65, regardless of gender and race. The search strategy will cover PICO. The quality of studies will be determined using Consort, STROBE, NHLBI, PEDro and CASP checklists in expert consensus.

Dissemination. The results of this plan will clarify which electrical stimulation current will improve pain and function in chronic non-specific low back pain. This is valuable in clinical practice to optimize therapeutic planning.
\end{abstract}

\section{KEY WORDS}

Electrical stimulation; current; pain; function; nonspecific chronic low back pain; physical therapy.

\section{INTRODUCTION}

Low Back Pain (LBP) is a common health issue all over the world which directly shakes the quality of life, daily activities and social roles (1-3).

Considering the long term systemic complications of medications esp. for some high risk populations such as elderlies and adolescents, nowadays people prefer non-medical strategies (4). Consequently, physical therapy is the non-invasive approach of choice in the treatment of CLBP (5). Electrical Stimulation (ES) currents may be administered as a part of physical therapy to eliminate pain (6).

ES currents in the CLBP has been studied widely in clinical trials $(7-12)$. The results are controversial due to variations in stimulation parameters, demographic and anthropometric characteristics of the participants, design of the study, the outcome measure, and intervention duration and planning. 
It seems that the impact of various types of ES currents on non-specific CLBP has not been reviewed yet. The question is which type of the therapeutic ES currents may help improving pain and function in subjects with chronic non-specific LBP? Present work is the protocol of a systematic review to determine the effect of different ES currents on pain and function in people suffering from chronic non-specific LBP.

\section{OBJECTIVES}

To determine the effect of various ES currents on the pain and function of people with non-specific chronic low back pain.

\section{MATERIALS AND METHODS}

\section{Trial eligibility criteria}

The inclusion/exclusion criteria have been defined to properly cover search strategies and PICOs. The detail of the criteria is summarized below.

\section{Study types}

All study types i.e. Clinical Trials, Cohort, Case-controls, Cross-sectionals, Observational Descriptive, Case Report, Case Series, Ecological Studies, Systematic Reviews and thesis and dissertation will be included. Qualitative Studies and Narrative Reviews will not take into quality assessment.

\section{Participants}

People with non-specific CLBP between 18-65 who were treated using ES currents regardless of gender and ethnicity. The study will be entered the review if the participants suffered from back pain for at least three months without known pathology. Studies on nonhuman samples, professional athletes, subjects with acute LBP, or the studies that included LBP cases with less than three months duration of symptoms will be excluded. LBPs of specified origin such as inflammatory diseases, spondylo-arthropathies, disk hernia, spinal canal/foraminal stenosis, visceral pains, fractures and trauma will not be eligible. If the participants complained from referral or radicular symptoms, the study will also be excluded. Pregnant women, children (under 18 years) and elderly (over 65 years) will not be of interest in present protocol.

\section{Interventions}

The intervention group shall receive ES currents including: Transcutaneous Electrical Nerve Stimulation (TENS),
Interferential (IF), Diadynamic, High-voltage, Russian currents, Faradic.

\section{Comparators}

Studies will be approved to be included only if there was a control group (without treatment), sham group (placebo treatment), healthy group (of matched healthy subjects) or if two or more ES currents were compared.

\section{Outcome measures}

Pain and function will be the main outcome measures in this systematic review; pain will be assessed by the Numerical Rating Scale (NRS), Visual Analogue Scale (VAS), Pressure Pain Threshold (PPT), MCGill Pain Questionnaire. Function will be measured by Roland-Morris Disability Questionnaire, Oswestry Disability Index, or clinical/functional tests. Other tools may be also considered according to the included studies.

Studies will be included the experimental (case) group and the control group were established and the related monitoring data were introduced. Two classification variables, continuous variables and variance test should be administered. Within-(pre-post) and between-group measures will be analyzed for clinical trials. For cohorts and case-controls odds ratio will be of interest. Effect size and confidence intervals will be of value in all types included studies.

Additional outcome measures will be considered upon progression of the study. Some of anticipated secondary outcome measures are: anthropometric data (weight, height, $\mathrm{BMI}$ ), psycho-social and cultural data (literacy level, marital status, economical class), comorbidities (diabetes, cardiac or pulmonary disorders, smoking, alcohol consumption).

\section{Search methods to identify studies}

Pubmed, ISI Web of Science, Scopus, Clinical Key, Science Direct, Medline, Embase, PEDro, ProQuest, the Cochrane Library, PROSPERO, and also $\mathrm{MOH}$ Thesis, $\mathrm{MOH}$ Articles, Magiran and SID as the national databases will be searched. Also Google Scholar search engine will be used. The review studies will not be included in the final analysis. However, their references will be checked through Cross Reference. The main key words will be electrical stimulation current, pain, function, nonspecific chronic low back pain. The key terms will be updated during the search process. The search strategy will cover the following search query and also PICO.

Nonspecific AND chronic AND ("low back pain" OR (low AND back AND pain) OR "back ache") AND ("electric 
stimulation*" OR (electric AND stimulation) OR "electrical stimulation*") AND (TENS OR "Transcutaneous Electrical Nerve Stimulation" OR Interferential OR Diadynamic OR High Voltage OR Russian OR Faradic) AND ("control group*" OR ((placebo or unrealistic) AND (treatment OR therapy*)) AND Function*) in TITLE/SUMMARY/ KEY WORDS.

P: nonspecific AND chronic AND "low back pain" OR (low AND back AND pain) OR "back ache").

I: ("electric stimulation*" OR (electric AND stimulation) OR "electrical stimulation*") AND (TENS OR "Transcutaneous Electrical Nerve Stimulation” OR Interferential OR Diadynamic OR High Voltage OR Russian OR Faradic).

C: "control group*" OR ((placebo or unrealistic) AND (treatment OR therapy)). O: function*.

The search procedure will be repeated every few weeks by two researchers (SIL and TSM) who are blind to other ones' findings. The researchers will also review and retrieve the reference list of all included articles (Hand Search). In the case of no access to the paper-based or electronic full text of an article, the authors (corresponding or first author) or editor of the publishing journal will be contacted thrice. In the case of unsuccessful tries, the article will be withdrawn from study. The Gray Literature will be search through their specified databases including "http://www.gateway.com/ worldwide/, "http://www.proquest.com/”, "http://www. irct.ir/", "http://www.trialscentral.com/".

\section{Study selection}

The articles that were published until the end of August 2019 (Shahrivar 9 $9^{\text {th }}, 1398$ Persian Calendar) will be acceptable. To ensure accuracy of the search results, the search will be extended three years before the publication date of the first article found for each type of ES currents. Search results and Reference lists will be imported to the

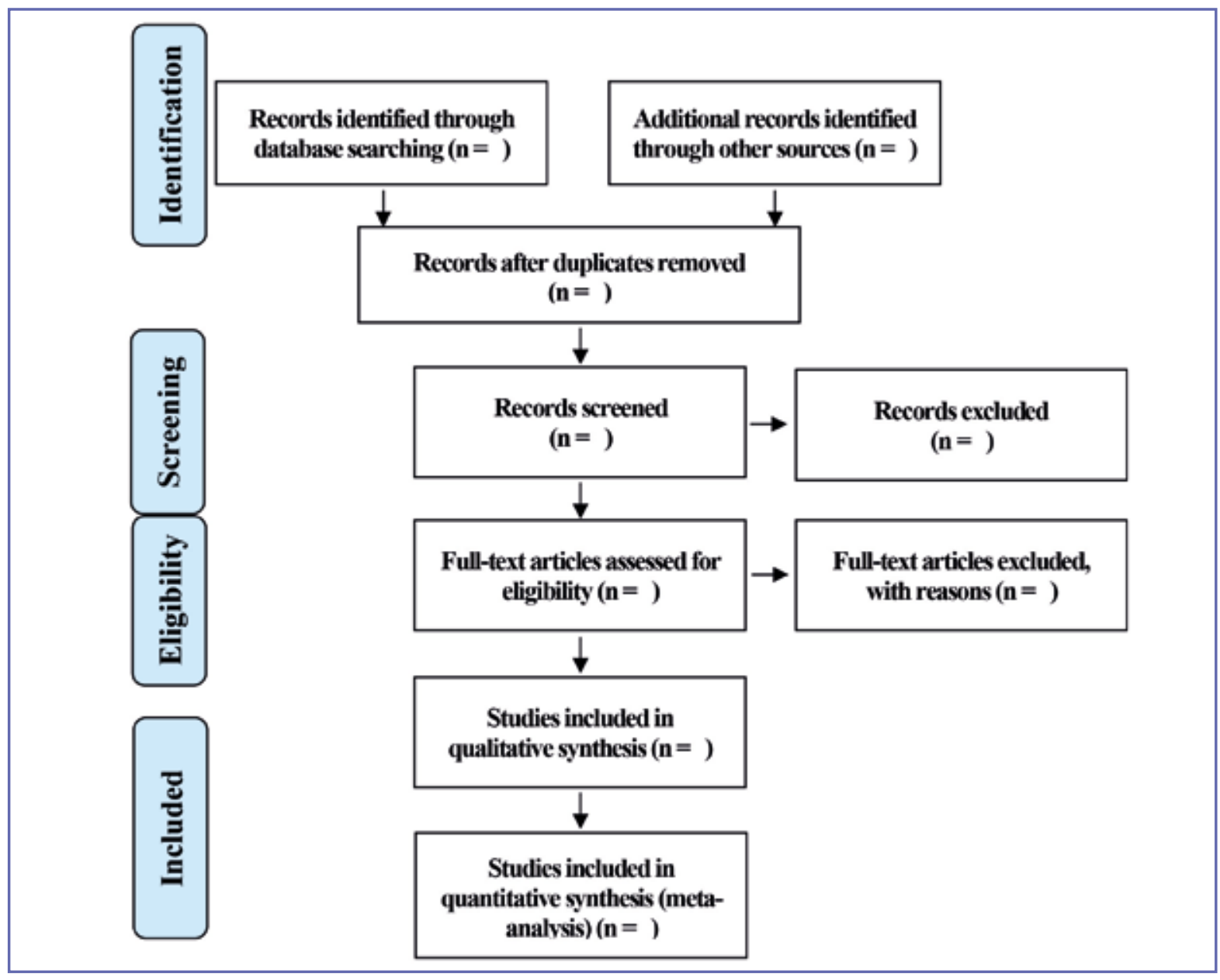

Figure 1. PRISMA 2009 Flow diagram of the articles selection process (14). 
citation manager software. Duplicates and unrelated articles will be removed through screening titles and summaries. Then, the full text of the remaining articles will be reviewed in detail. After excluding irrelevant articles, and those that fail according to inclusion criteria, the quality of the remaining articles will be criticized and evaluated. All stages will be performed by two independent researchers (SIL and TSM) under the supervision of ZSR, FB and AR. The principal investigator will supervise the procedure. Any disagreement will be resolved through consensus. The entire process of study selection is summarized in the PRISMA flow diagram

\section{Data extraction}

The Search will be done on aforementioned databases. Screening of PubMed title/abstract will be done by two researchers (SIL and TSM) to calculate inter-rater agreement under the supervision of ZSR, AR and FB. The qualitative studies and narrative reviews will not be included in the final analysis while their references will be checked through Cross Referencing. Key terms will be updated along study progression. The references of included articles will be checked manually (Hand Search). If the full text of any article was not found, the corresponding/ first author or the editor of the publishing journal will be contacted thrice. If not successful, the article will be withdrawn.

The screening of the title/summaries will be carried out independently by SIL and TSM. The total number of the extracted articles from each database will be reported in a flowchart. Unrelated and duplicate articles will be excluded. All the search results will be saved in a citation manager. This screening process will be repeated again from the beginning every three months to justify fast exclusion phase.

Remaining articles will be criticized through review of fulltext article by SIL, ZSR, FB, AR and TSM independently. Decisions will be made regarding inclusion/exclusion criteria. Reasons for the exclusion will be reported.

Data from the approved full texts will be entered into Excel sheet (data extraction) that covers publication details (the author(s), title, publication year, journal, country), participants, study design, sample size, randomization, allocation concealment, blinding, intervention, control intervention, main outcomes, adverse effects, follow-up, withdrawals and results. Necessary information will be obtained through correspondence with the original authors in case. PI (ZSR) supervises the procedure. Disagreements in every step will be clarified through consensus.

\section{Quality assessment}

Considering study design, included articles will be qualified in expert consensus using Consort, STROBE, PEDro, CASP and NHLBI checklists. The score for each checklist will be recorded. Articles that gain $50 \%$ of total score of one of the checklists will be considered for quantitative analysis. The articles' quality will be ranked as high $(75 \%)$, medium $(50-75 \%)$, low $(25-50 \%)$, poor $(<25 \%)$ according to the scores they receive using each single checklist.

PEDro scale will be used to assess the validity of selected studies. The checklist consists of 11 items that may be marked as a "plus" (when the item has been properly addressed in the article text) or "minus" (if the item cannot be localized throughout the text) (14).

The standard STROBE checklist, which has 22 items, evaluates the quality of case-control studies and cohort in two independent scales $(15,16)$. However, some researchers recommend Study Quality Assessment Tools proposed by National Heart, Lung and Blood Institute (NHLBI) as more precise qualification instrument for observational studies (17). Therefore, these articles will also be evaluated using NHLBI recommended checklists.

The CONSORT checklist has been introduced as an international standard and a standardized approach to clinical practice report (18). Studies that earn a score of $50 \%$ or more will be included in the study. For precise assessment of the articles, appropriate CONSORT extension may be administered (19).

CASP checklist has been specifically adapted for various study designs including case-control studies, cohort and clinical trials (20). Articles that earn a score of $50 \%$ or more will be included in the study.

For comprehensive qualification of all article types that will meet the inclusion criteria of the study, TIDieR checklist will be administered beside the main checklist.

\section{Measure of Treatment Effects}

In the case of measuring continuous outcomes i.e. pain scales, the mean difference (MD) with a $95 \%$ CI will be analyzed, and other form of reports will be covert into MD. For dichotomous data (eg, adverse events), a risk ratio (RR) with a $95 \% \mathrm{CI}$ and for other binary data an $\mathrm{RR}$ value will be calculated.

\section{Missing data}

Missing data will be collected through contacting the research team. In case of no adequate reply the available data will be analyzed solely. 


\section{Statistical Methods}

\section{Data synthesis}

If possible, the random-effects or fixed-effects model will be developed for the meta-analysis Using RevMan (Review Manager Software, Version 5.3; Copenhagen: The Nordic Cochrane Centre, The Cochrane Collaboration, 2014, The Cochrane Collaboration, Oxford, England). The RR with the $95 \%$ CI for dichotomous data and the MD with the $95 \%$ CI for continuous data will be calculated if possible. In the case of acceptable heterogeneity $\left(\mathrm{I}^{2} \leq 50 \%\right)$, the RR and MD will be calculated in the fixed-effects model; otherwise, the random-effects model will be administered. If quantitative synthesis is not achievable, the results will be reported in the narrative description

\section{Assessment of heterogeneity}

Heterogeneity will be determined by the $\mathrm{I}^{2}$ and $\chi^{2}$ tests. Considering $50 \%$ as the cut-off for statistical analysis of $\mathrm{I}^{2}$, the meta-analysis will be indicated in the case of significant heterogeneity of $\mathrm{I}^{2}>50 \%$, and a subgroup analysis to explore the possible causes will be of value.

\section{Subgroup analysis}

Subgroup analysis including the analysis of electrical parameters of each current type, type of control, countries and different outcomes will be performed to assess the heterogeneity between the studies.

\section{Sensitivity analysis}

If the heterogeneity persists following subgroup analysis or if there were studies with imperfect results, the sensitivity analysis will be developed by removing the lower quality studies. Then, the meta-analysis will be run again and the results of the two meta-analyses will be compared and discussed according to the sample size, strength of evidence and influence on the pooled effect size.

\section{Assessment of reporting biases}

If enough studies were included for qualitative analysis (at least 10 trials for each current), the publication bias will be assessed by funnel plots. In addition, the impacts of possible selective reporting, reporting deviations from the original protocols, effect of protocol compliance and adherence will be discussed.

\section{DISCUSSION}

The study included all existing articles of any type published any time concerning the application of ES currents in non-specific LBP. Therefore, based on the results of this study, it can be specified how various types of ES currents may improve pain and function in people suffering non-specific CLBP. The results of this study may be cited in the setting of clinical guidelines, legal tariffs for treatment and standard planning of physical therapy sessions. The results will also help physical therapists to decide about low risk and low cost electric stimulus currents with the optimal therapeutic outcome when managing of non-specific CLBP. In the case of reaching enough studies through which various currents were compared, the most effective current for CLBP clients will be identifiable. With regard to the strict but comprehensive selection criteria, the included studies may provide detailed information about parameters and durability of the best-practice ES currents. The studies will be categorized and apprised using various checklist in a peers' consensus to assure high internal validity of article scoring phase. If enough cost-effectiveness study retrieved, the results will also provide field applicable basis for financial preference of selecting electrical stimulus currents in non-specific CLBP. The search has been already started and the preliminary data shows that in spite of clinical use of some ES currents, they have no scientific research background concerning practical parameter setting, clinical efficacy or durability of the sedative or functional effects; that means that the results will highlight existing research and clinical gaps to design further studies. Development of a meta-analysis will be possible in case of enough homogeneous articles in each field.

\section{ETHICS}

The study has been funded and ethically approved by Isfahan University of Medical Sciences (Ethics Code: IR.MUI. REC.1397.090) as a part of a thesis for Master's Degree in Physical Therapy by Sepideh Izadi Laybidy (Registration code: 297051). The sponsor has no role in data collection, analysis of the data and drafting the manuscript. The study meets the ethical standards of the journal of Muscle, Ligament, and Tendon Journal (21).

\section{ACKNOWLEDGMENTS}

This study will be developed with the financial support and ethically approval by Isfahan University of Medical Sciences (Ethics Code: IR.MUI.REC.1397.090) as a part of a thesis for Master's Degree in Physical Therapy by Sepideh Izadi Laybidy (Registration code: 297051). The protocol has been registered in International Prospective Register of Systematic Reviews (CRD42019121819). The sponsor will not play a role in data collection, analysis of the data and drafting the manuscript. 


\section{CONFLICT OF INTERESTS}

The authors declare that they have no conflict of interests.

\section{REFERENCES}

1. Buchmuller A, Navez MF, Milletre-Bernardin MF, et al. Value of TENS for relief of chronic low back pain with or without radicular pain. EJP 2011-16:656-665.

2. Comachio J, Oliveira Magalhaes M, Nogueira Burke T, et al. Efficacy of acupuncture and electroacupuncture in patients with nonspecific low back pain: study protocol for a randomized controlled trial. Trials 2015;16:469.

3. Correa JB, Costa LO, Oliveira NT, Lima WP, Sluka KA, Liebano RE. Effects of the carrier frequency of interferential current on pain modulation and central hypersensitivity in people with chronic nonspecific low back pain: a randomized placebo-controlled trial. EJP 2016;20:1653-1666.

4. Yokoyama M, Sun X, Oku S, et al. Comparison of percutaneous electrical nerve stimulation with transcutaneous electrical nerve stimulation for long-term pain relief in patients with chronic low back pain. Anesth Analg 2004;98:1552-6.

5. Bhadauria EA, Gurudut P. Comparative effectiveness of lumbar stabilization, dynamic strengthening, and Pilates on chronic low back pain: randomized clinical trial. JER 2017;13:477-85.

6. Rajfur J, Pasternok M, Rajfur K, et al. Efficacy of Selected Electrical Therapies on Chronic Low Back Pain: a Comparative Clinical Pilot Study. Med Sci Monit 2017;23:85-100.

7. Adedoyin RA, Olaogun MOB, Onipede TO, Ikem IC, Egwu $\mathrm{MO}$, Bisiriyu LA. Effects of different swing patterns of interferential currents on patients with low back pain: a single control trial. Fiz Rehab 2005-16:61-66.

8. Albornoz-Cabello M, Maya-Martin J, Dominguez-Maldonado G, Espejo-Antunez L, Heredia-Rizo AM. Effect of interferential current therapy on pain perception and disability level in subjects with chronic low back pain: a randomized controlled trial. Clin Rehabil 2017;31:242-9.

9. Elserty N, Kattabei O, Elhafez H. Effect of Fixed Versus Adjusted Transcutaneous Electrical Nerve Stimulation Amplitude on Chronic Mechanical Low Back Pain. J Altern Complement Med 2016;22:557-562.
10. Itoh K, Itoh S, Katsumi Y, Kitakoji H. A pilot study on using acupuncture and transcutaneous electrical nerve stimulation to treat chronic non-specific low back pain. Complement Ther Clin Pract 2009;15:22-5.

11. Jarzem PF, Harvey EJ, Arcaro N, Kaczorowski J. Transcutaneous electrical nerve stimulation (TENS) for short term treatment of low back pain: randomized double blind crossover study of sham versus conventional TENS. JMP 2005.

12. Topuz O, Ozfidan E, Ozgen M, Ardic F. Efficacy of transcutaneous electrical nerve stimulation and percutaneous neuromodulation therapy in chronic low back pain. J Back Musculoskelet Rehabil 2004; 17:127-133.

13. Moher D, Liberati A, Tetzlaff J, Altman DG. Preferred reporting items for systematic reviews and meta-analyses: the PRISMA statement. PLoS Med 2009;62:1006-1012.

14. Maher CG, Sherrington C, Herbert RD, Moseley AM, Elkins M. Reliability of the PEDro scale for rating quality of randomized controlled trials. Phys Ther 2003;83:713-21.

15. Vandenbroucke JP, von EE, Altman DG, et al. Strengthening the Reporting of Observational Studies in Epidemiology (STROBE): explanation and elaboration. Int J Surg 2014;12:1500-24.

16. von EE, Altman DG, Egger M, Pocock SJ, Gotzsche PC, Vandenbroucke JP. The Strengthening the Reporting of Observational Studies in Epidemiology (STROBE) statement: guidelines for reporting observational studies. Int J Epidemiol 2007;18:800-4.

17. da Costa BR, Cevallos M, Altman DG, Rutjes AW, Egger M. Uses and misuses of the STROBE statement: bibliographic study. BMJ Open 2011;1:-000048.

18. Moher D, Hopewell S, Schulz KF, et al. CONSORT 2010 explanation and elaboration: updated guidelines for reporting parallel group randomised trials. BMJ 2010;340:698-702.

19. Consort group. http://www.consort-statement.org/extensions. 2019.

20. Oxford Centre for Triple Value Healthcare Ltd (3V) portfolio. The Critical Appraisals Skills Programme (CASP). Oxford Centre for Triple Value Healthcare Ltd (3V) portfolio 2020.

21. 21. Padulo J, Oliva F, Frizziero A, Maffulli N. Muscles, Ligaments and Tendons Journal. Basic principles and recommendations in clinical and field Science Research: 2018 update. MLTJ 2018; 8(3): 305-7. 